\title{
Bifurcation Analysis of Discrete-Time Systems with Multiple Time-Delays
}

\author{
Li Zeng, ${ }^{1, *}$ \\ ${ }^{1}$ School of Finance, Guangdong University of Foreign Studies \\ Guangzhou, Guangdong 510006, P. R. China \\ Corresponding author
}

\begin{abstract}
A unified frequency-domain approach to analyze Neimark-Sacker bifurcations and flip bifurcations of discrete time systems with multiple time-delays in the linear feed-forward and the nonlinear term is presented. The technique relies on the harmonic balance approach (HBA) and feedback systems theory. The expressions of the bifurcation solution and the stability are derived. One example is used to verify the result obtained.
\end{abstract}

Keywords - discrete-time systems; harmonic balance approach; neimark-sacker bifurcation; flip bifurcation

\section{INTRODUCTION}

Bifurcation theory is a classical mathematical subject and also a very attractive research topic in many disciplines such as the physics, mechanics, chemical, biomedical and engineering systems etc. The Neimark-Sacker bifurcation occurs under assumptions that are generic, i.e. robust to perturbations. It has been observed in various applications, such as power electronics, biology, economics, neural sciences, numerical analysis, neural networks and adaptive control. For discretetime systems, this bifurcation is sometimes called the Hopf bifurcation for map. In the continuous-time setting, the Neimark-Sacker bifurcation is often equated with the secondary Hopf bifurcation, which involves using a Poincaré map to discretize the continuous system. A flip bifurcation is also called the period-doubling bifurcation for map. It is a bifurcation in which the system switches to a new behavior with twice the period the original system. That is, there exists two points such that applying the dynamics to each of the points yields the other point. Flip bifurcations can also occur in continuous dynamical systems, namely when a new limit cycle emerges from an existing limit cycle, and the period of the new limit cycle is twice that of the old one. For purposes of mathematical analysis, the Neimark-Sacker bifurcation and the flip bifurcation are most conveniently considered in the discrete-time context. Most of the present literatures about the research on the Neimark-Sacker bifurcation and the flip bifurcation are based on the methods in the time domain either in the continuous-time system or in the discrete-time system, especially in the latter.

The harmonic balance approach (HBA) is a classical technique for studying nonlinear system oscillations. The main advantage of HBA is that it involves algebraic equations with less unknowns than the methods in the time domain, for problems for which low orders of approximation are sufficient to obtain an accurate solution. For this reason, in recent years, much interest has devoted to the study of the bifurcations and chaos in nonlinear dynamical systems based on HBA. This technique has been employed in the context of continuous dynamical systems (see. [1]-[5] and the references in these text). But in the context of the discrete dynamical systems, there are relatively less contributions done in the literature. D'amico, Moiola and Paolini discuss Neimark-Sacker bifurcations and flip bifurcations of the nonlinear maps in a unified frequencydomain approach (FDA) in [6]-[8]. The method relies on HBA and feedback systems theory in the frequency domain. Zeng, Zhao, Sun and Zong analyze the Neimark-Sacker and flip bifurcations of maps with delay in the linear term via the frequency-domain method in [9].

The objective of this paper is to extends the results for maps with one delay in the linear term stated in [9] to the counterpart of the maps with multiple time-delays in the linear feedforward and the nonlinear term. This paper is organized as follows. In section 2, HBA results are obtained for the prediction and stability analysis of Neimark-Sacker and flip bifurcations. Section 3 contains a detailed example illustrating analysis. Conclusions are contained in section 4.

\section{HARMONIC BALANCE APPROACH IN NONLINEA R MAPS WITH MULTIPLE TIME-DELA YS}

In this paper, we consider the following discrete-time systems with multiple time-delays:

$$
\begin{gathered}
x(k+1)=A_{0} x(k)+\sum_{t=1}^{N} A_{t} x\left(k-\tau_{t}\right)+B g\left(y\left(k-\tau_{N+1}\right) ; \mu\right) \\
y(k)=C x(k)
\end{gathered}
$$

where $k \in \mathrm{N}, \quad x(k) \in \mathrm{R}^{n}$ is the state vector of the system, $\mu \in \mathrm{R}^{s} \quad$ is the system bifurcation parameter-vector, $g(\cdot, \cdot): \mathrm{R}^{m} \times \mathrm{R}^{s} \rightarrow \mathrm{R}^{l}$ can be viewed as a system input, $y(k) \in \mathrm{R}^{m}$ is the system output, $A_{t} \in \mathrm{R}^{n \times n}(t=0,1,2, \cdots, N)$, $B \in \mathrm{R}^{n \times l}, C \in \mathrm{R}^{m \times n}$, all matrices are smooth functions of $\mu$, $\tau_{t}>0(t=1,2, \cdots, N, N+1)$ are the time-delay parameters.

According to [10], there are many different but equivalent feedback representations for (1). This can be easily seen by introducing an arbitrary matrix $D \in \mathrm{R}^{l \times m}$ (which may also depend on $\mu$ ) and rewriting (1) as 


$$
\begin{gathered}
x(k+1)=\left(A_{0}+B D C\right) x(k)+\sum_{t=1}^{N} A_{t} x\left(k-\tau_{t}\right)+B u(k) \\
y(k)=C x(k)
\end{gathered}
$$

where $u(k)=g\left(y\left(k-\tau_{N+1}\right) ; \mu\right)-D y(k)$.

Taking z-transform in (2) with zero-initial conditions, yields the following representation in the frequency domain

$$
Z(e)=-G(z ; \mu) Z(u)
$$

where

$$
\begin{gathered}
e(k)=-y(k)=-C x(k) \\
G(z ; \mu)=C\left[z I-\left(A_{0}+B D C\right)-\sum_{t=1}^{N} A_{t} z^{-\tau_{t}}\right]^{-1} B \\
u(k)=f\left(e\left(k-\tau_{N+1}\right) ; \mu\right)=g\left(-e\left(k-\tau_{N+1}\right) ; \mu\right)+D e(k) .
\end{gathered}
$$

This representation suggests that the system (2) can be thought as the feedback interconnection, where the linear system $G(\cdot ;)$ and the nonlinear map $f(\cdot ; \cdot)$ with time-delays are defined as above.

From (3), the original problem can be solved for the variable $e$ (contained in $Z(\cdot))$ in the frequency domain. So if $\hat{x}$ is a fixed point of (1), $\hat{e}=-C(\mu) \hat{x}$ can be considered as a fixed point in the frequency domain. Based on control theory, the fixed point $\hat{e}$ can be obtained by solving the nonlinear equation

$$
\hat{e}=-G(1 ; \mu) f(\hat{e} ; \mu)
$$

where $G(1 ; \mu)=C\left[I-\left(A_{0}+B D C\right)-\sum_{t=1}^{N} A_{t}\right]^{-1} B$. And the linearization of the nonlinear feedback function about $\hat{e}$ gives

$$
J(\mu) z^{-\tau_{N=1}}+D=\left.\frac{\partial g(-e ; \mu)}{\partial e}\right|_{e=\hat{e}} z^{-\tau_{N=1}}+D .
$$
by

The characteristic equation of the linearized system is given

$$
h(\lambda, z ; \mu)=\operatorname{det}\left[\lambda I-G(z ; \mu)\left(J(\mu) z^{-\tau_{N+1}}+D\right)\right]=0
$$

The critical points $\left(\omega_{0}, \mu_{0}\right)$ can be deduced from $h\left(-1, e^{i \omega} ; \mu\right)=0$.

From the generalized Nyquist stability criterion, we can deduce that the crossing of an eigenvalue of (1) in the time domain through the unit circle is replaced by the crossing of one eigenvalue of $G\left(e^{i \omega} ; \mu\right)\left(J(\mu) e^{-i \omega_{x+1}}+D\right)$, denoted as $\hat{\lambda}\left(e^{i \omega} ; \mu\right)$, over the critical point $-1+i 0$ for certain frequency $\omega_{0}$ in the frequency domain. Fixing $\mu=\tilde{\mu}$ and varying $\omega$, the locus of the eigenvalue or "eigenlocus" is obtained. When there is a Neimark-Sacker bifurcation, this locus is analyzed for
$\omega_{0}=\omega_{H} \neq 0$ excluding the strong resonance cases $e^{i \omega_{H}}=1$ for $r=1,2,3,4$. Flip bifurcations are distinguished by $\omega_{0}=\omega_{D}=\pi$. While a unit eigenvalue, which is a necessary condition for static bifurcation, is associated with an eigenlocus that crosses the point $-1+i 0$ at $\omega_{0}=0$.

For simplicity, let $J=J(\mu), \quad G\left(e^{i i_{\nu}}\right)=\left.G(z ; \mu)\right|_{z=e^{i \nu}}$, $\hat{\lambda}\left(e^{i \omega}\right)=\hat{\lambda}\left(e^{i \omega} ; \mu\right)$ also can be seen as a function of $\tau_{t}(t=1,2, \cdots, N, N+1)$. Let us fix $\mu$ close to $\mu_{0}$, then the eigenlocus of $\hat{\lambda}\left(e^{i \omega}\right)$ lies near the critical point $-1+i 0$. If a periodic solution exists, it can be written as the $2 q$-order approximation

$$
e(k)=\hat{e}+\operatorname{Re}\left\{\sum_{r=0}^{2 q} E^{r} e^{i r u k}\right\}
$$

where $\operatorname{Re}\{\cdot\}$ denotes the real part of a complex number, $\omega$ is the fundamental frequency of the periodic solution, and $E^{r}$ is a complex number in the $r$-th order harmonic of the expansion.

Expanding the nonlinear function $f(\cdot ;)$ with respect to $e(k)$ in Taylor series up to the $2 q+1$-order, i.e.

$$
\begin{aligned}
f(x+h)= & f(x ; \mu)+(D f)_{x} h+\frac{1}{2 !}\left(D_{2} f\right)_{x} h \otimes h \\
& +\cdots+\frac{1}{j !}\left(D_{j} f\right)_{x} \otimes^{j} h+\mathcal{O}\left(\otimes^{j+1} h\right)
\end{aligned}
$$

where $\otimes$ is the standard tensor product operator, $\left(D_{k} f\right)_{x}$ can be viewed as the linear operator of the tensor-product space $\otimes^{k} R^{m}$. And then replacing $e(k)$ with (6), we can obtain

$$
f\left(e\left(k-\tau_{N+1}\right) ; \mu\right)=f(\hat{e} ; \mu)+\operatorname{Re}\left\{\sum_{r=0}^{2 q} F_{d}^{r} e^{i r_{*} k}\right\},
$$

where $F^{r}$ is a complex number in the $r$-th order harmonic of the expansion, which satisfies

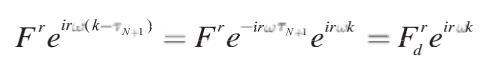

Here " $d$ " is used to indicate time-delayed quantities. Equating the output of the system with the input of the linear plant, we derive the harmonic balance equations, namely,

$$
E^{r}=-G\left(e^{i r_{*}}\right) F_{d}^{r} \quad r=0,1, \cdots, 2 q .
$$

\section{A. Neimark-Sacker Bifurcation}

For analysis of Neimark-Sacker bifurcation and simplicity, let $q=1$. By using the Taylor expansion of $f(e(k) ; \mu)$ at $\hat{e}$ and (7), the relation of $E^{r}$ and $F_{d}^{r}$ is the following equation 


$$
\begin{aligned}
E^{0}= & -G(1)\left[(J+D) E^{0}+\frac{1}{4} D_{2} g\left(E^{1} \otimes \bar{E}^{1}\right)\right]+\mathcal{O}\left(\left|E^{1}\right|^{4}\right) \\
E^{1}= & -G\left(e^{i \nu}\right)\left[\left(J e^{-i \omega T_{N+1}}+D\right) E^{1}\right. \\
& +1 / 2 \cdot D_{2} g\left(2 E^{0} \otimes E^{1} e^{-i \tau_{N+1}}+\bar{E}^{1} \otimes E^{2}\right) e^{-l \omega T_{N+1}} \\
& \left.+1 / 6 \cdot D_{3} g\left(3 / 4 E^{1} \otimes E^{1} \otimes \bar{E}^{1} e^{-i_{N N+1}}\right)\right]+\mathcal{O}\left(\left|E^{1}\right|^{4}\right) \\
E^{2}= & -G\left(e^{i 2 \omega}\right)\left[\left(e^{-i \omega N+1}+D\right) E^{2}\right. \\
& \left.+1 / 4 D_{2} g\left(E^{1} \otimes E^{1} e^{-i 2 \omega_{N+1}}\right)\right]+\mathcal{O}\left(\left|E^{1}\right|^{4}\right)
\end{aligned}
$$

where $D_{j} g=\left.D_{j} g(e ; \mu)\right|_{e-\hat{e}}$. Similar as in [10], these equations are solved in terms of $E^{1}=\theta v$, where $v$ is the normalized right eigenvector associated with the eigenvalue $\hat{\lambda}\left(e^{i \omega}\right)$ (the eigenvalue $\lambda$ satisfying (5) that is closest to the critical point $-1+i \cdot 0)$, and $\theta$ is a measure of the amplitude of the periodic solution. The other complex vectors are given by $E^{0}=V_{0 H} \theta^{2}$ and $E^{2}=V_{2 H} \theta^{2}$ with

$$
\begin{aligned}
& V_{0 H}=-1 / 4 H(1 ; \mu) Q \bar{v} \\
& V_{2 H}=-1 / 4 H\left(e^{i 2 \omega} ; \mu\right) Q v e^{-i 2 \omega T_{N+1}}
\end{aligned}
$$

where $\quad H(z ; \mu)=\left[I+G(z ; \mu)\left(J(\mu) z^{-\tau_{N+1}}+D\right)\right]^{-1} G(z ; \mu) \quad$, $Q=\left(D_{2} g\right) v$ can be computed as in [9].

So, for the periodic solution (1) to exist, it is necessary that

$$
\hat{\lambda}\left(e^{i_{\omega}} ; \mu\right)=-1+\xi_{H d} \theta^{2}+\mathcal{O}\left(\theta^{3}\right)
$$

with

$$
\xi_{H d}=-\frac{s^{T} G\left(e^{i \omega}\right) p_{H d}}{s^{T} v}
$$

Where $p_{H d}(\omega, v, \tau)=\left[Q V_{0 H}+1 / 2 \bar{Q} V_{2 H}+1 / 8 L \bar{v}\right] e^{-i \omega T_{N+1}}, \quad s$ is the normalized left eigenvector associated with the eigenvalue $\hat{\lambda}\left(e^{i \omega}\right), L=\left(D_{3} g\right) v^{2}$.

Similar as in [9], we can prove that the stability of the emerging periodic solution can be determined by the stability index $\sigma_{H d}$, i.e.

$$
\sigma_{H d}=\operatorname{Re}\left\{\frac{s^{T} G\left(e^{i \omega}\right) p_{H d} e^{-i \omega}}{\left.s^{T} \frac{\partial}{\partial z}\left[G(z ; \mu)\left(J(\mu) z^{-\tau_{N=1}}+D\right)\right]\right|_{z=e^{e^{\omega}}} v}\right\}
$$

If $\sigma_{H d}>0\left(\sigma_{H d}<0\right)$, there experiences the supercritical (subcritical) Neimark-Sacker bifurcation and the emerging invariant circle is stable(unstable). Case with $\sigma_{H d}=0$ has been discussed in $[8,11]$.

The following is the proof of the stability index $\sigma_{H d}$. Supposing that the matrix $G(z ; \mu)\left(J(\mu) z^{-\tau_{N+1}}+D\right)$ has an eigenvalue $\hat{\lambda}\left(z_{0} ; \mu_{0}\right)=-1+i \cdot 0$, it is easy to verify that the matrix $G(z ; \mu)\left(J(\mu) z^{\tau_{w a 1}}+D\right)$ has the same eigenvalue. Moreover, at the critical value $\mu=\mu_{0}, \omega=\omega_{0}$, the following hold: $z_{0}=e^{i \omega_{0}}, \bar{z}_{0}=e^{-i \omega_{0}}$, which are exactly the two corresponding complex eigenvalues of (1) of the discrete-time version of the HBA for a given value $\mu=\mu_{0}$ according with the NeimarkSacker bifurcation.

Without lose of generality, supposing that when $\mu>\mu_{0}$, the pair of complex conjugate eigenvalues of (9) crosses the unit circle, i.e. $z=\rho e^{i \omega}(\rho>1)$. What's more, considering that under this condition, the analysis in the frequency domain guarantees the existence of a periodic solution with an approximate frequency $\hat{\omega}$ and a small amplitude $\theta$. Then we can write

$$
\begin{aligned}
& G\left(e^{i \dot{i}} ; \mu\right)\left(J(\mu) e^{-\dot{i}_{x+2}}+D\right)=G(z ; \mu)\left(J(\mu) z^{-\tau_{x+1}}+D\right) \\
& +\left(e^{i j}-z\right) \frac{d}{d z}\left[G(z ; \mu)\left(J(\mu) z^{x_{x=1}}+D\right)\right] \\
& +\frac{1}{2 !}\left(e^{i=}-z\right)^{2} \frac{d^{2}}{d z^{2}}\left[G(z ; \mu)\left(J(\mu) z^{r_{x y}}+D\right)\right]+\cdots
\end{aligned}
$$

Substituting (7) into the second equation of (8), and letting $E^{1}=\theta(v+w)$, then we can derive

$$
\begin{aligned}
& {\left[G\left(e^{i \hat{\omega}} ; \mu\right)\left(J(\mu) e^{-i \hat{\hat{\omega} \tau} \tau_{N+1}}+D\right)+I\right](v+w)} \\
& =-G\left(e^{i \hat{\omega}} ; \mu\right) p_{H d}(\hat{\omega}, v+w, \tau) \theta^{2}+\mathcal{O}\left(\theta^{3}\right)
\end{aligned}
$$

First multiplying both sides $s^{T}$, then substituting (11) into the equation and considering $w=\mathcal{O}\left(\theta^{2}\right)$, so we can get

$$
\begin{aligned}
& s^{T}\left[\left(e^{i \hat{\omega}}-z\right) \frac{d}{d z}\left[G(z ; \mu)\left(J(\mu) z^{-\tau_{N+1}}+D\right)\right]+\cdots\right] v \\
& =-s^{T} G\left(e^{i \hat{i}} ; \mu\right) p_{H d}(\hat{\omega}, v, \tau) \theta^{2}+\mathcal{O}\left(\theta^{3}\right)
\end{aligned}
$$

Observing that $e^{i \hat{\omega}}$ is in the small neighborhood of $e^{i \omega}$, So

$$
z-e^{i \hat{\omega}}=\gamma \theta^{2}+\mathcal{O}\left(\theta^{3}\right),
$$

where

and

$$
\gamma=\frac{s^{T} G\left(e^{i \nu} ; \mu\right) p_{H d}(\omega, v, \tau)}{\left.s^{T} \frac{d}{d z}\left[G(z ; \mu)\left(J(\mu) z^{-\tau_{v-1}}+D\right)\right]\right|_{z=e^{i e^{2}}} v}
$$

$$
\rho-\operatorname{Re}\left\{e^{i(\hat{\omega}-\omega)}\right\}=\operatorname{Re}\left\{\gamma e^{-i \omega}\right\} \theta^{2}+\mathcal{O}\left(\theta^{3}\right) .
$$

So for a small amplitude $\theta^{2}>0$, the necessary condition for which the above equation has a solution is that $\sigma_{H d}=\operatorname{Re}\left\{\gamma e^{-i \omega}\right\}$ and $\rho-\operatorname{Re}\left\{e^{i(\hat{\omega}-\omega)}\right\}$ have the same sign. Here $\sigma_{H d}$ is the stability index. If $\rho>1, \sigma_{H d}>0$. What's more, the fixed point become 
unstable and the stable closed invariant circle is bifurcating from the fixed point when $\mu$ is near to $\mu_{0}$ and $\mu>\mu_{0}$. In the same way, we can prove the following results: (i) If $\rho<1$, $\sigma_{H d}<0$. (ii) The unstable closed invariant circle is bifurcating from the fixed point when $\mu$ is near to $\mu_{0}$ and $\mu<\mu_{0}$.

\section{B. Flip Bifurcation}

As for analysis of the flip bifurcation, let $q=1$ and $\omega=\omega_{D}=\pi$. Then the period solution emerging from the flip bifurcation can be written as

$$
e(k)=\hat{e}+E^{0}+E^{1} e^{i \hbar k},
$$

and the harmonic balance equations can be derived as

$$
E^{0}=-G(1 ; \mu) F_{d}^{0}, \quad E^{1}=-G\left(e^{i \pi} ; \mu\right) F_{d}^{1}
$$

where

$$
\begin{aligned}
F_{d}^{0}= & (J+D) E^{0}+\frac{1}{2} D_{2} g\left(E^{1} \otimes E^{1} e^{-i 2 \pi \tau_{N+1}}\right)+\mathcal{O}\left(\left|E^{1}\right|^{4}\right) \\
F_{d}^{1}= & \left(J e^{-i \pi \tau_{N+1}}+D\right) E^{1}+D_{2} g\left(E^{0} \otimes E^{1} e^{-i \pi \tau_{N-1}}\right) \\
& +1 / 6 D_{3} g\left(E^{1} \otimes E^{1} \otimes E^{1} e^{-i 3 \pi \tau_{N+1}}\right)+\mathcal{O}\left(\left|E^{1}\right|^{4}\right)
\end{aligned}
$$

Analyzing the flip bifurcation as above section II-A, then the eigenvalue $\hat{\lambda}\left(e^{i \omega}\right)$ satisfies

$$
\hat{\lambda}(-1 ; \mu)=-1+\xi_{D} \theta^{2}+\mathcal{O}\left(\theta^{3}\right)
$$

and the stability of the emerging periodic solution can be determined by the stability index $\sigma_{D d}$

$$
\sigma_{D d}=\operatorname{Re}\left\{-\frac{s^{T} G\left(-1 ; \mu_{0}\right) p_{D d}(v, \tau)}{\left.s^{T} \frac{\partial}{\partial z}\left[G\left(z ; \mu_{0}\right)\left(J\left(\mu_{0}\right) z^{-T_{N+1}}+D\right)\right]\right|_{z=-1} v}\right\}
$$

where

$$
\begin{aligned}
& p_{D d}(v, \tau)=\left(Q V_{0 D}+1 / 6 L v e^{-i 2 \pi \tau_{N=1}}\right) e^{-i \pi \tau \tau_{N+1}} \\
& \xi_{D}=-\frac{s^{T} G(-1 ; \mu) p_{D d}(v, \tau)}{s^{T} v}
\end{aligned}
$$

$\left(\pi, \mu_{0}\right)$ satisfies $h\left(-1, e^{i \pi} ; \mu_{0}\right)=0$. If $\sigma_{D d}>0\left(\sigma_{D d}<0\right)$, there experiences the supercritical (subcritical) flip bifurcation and the emerging invariant circle is stable(unstable). Case with $\sigma_{D d}=0$ has been discussed by using normal form theory and singular theory in [12].

\section{EXAMPLE}

Consider the difference equation with two time-delays

$$
x(k+1)=\mu x(k)+a x\left(k-\tau_{1}\right)+b x\left(k-\tau_{2}\right)-\mu x^{2}\left(k-\tau_{3}\right)
$$

where $\tau_{i}(i=1,2,3)$ are integer time-delays, $0<a, b<1$. Here we only consider $b=2 a, \tau_{1}=1, \tau_{2}=2$ for simplicity. At the fixed point $\hat{x}=1+\frac{a+b-1}{\mu}=1+\frac{3 a-1}{\mu}$ of (19), firstly considering the Neimark-Sacker bifurcation for $1 / 3<a<1 / 2$. Selecting $B=C=1, D=0$, then

$$
\begin{gathered}
A_{0}=\mu, A_{1}=a, A_{2}=b=2 a, \\
f\left(e\left(k-\tau_{3}\right) ; \mu\right)=-\mu e^{2}\left(k-\tau_{3}\right), \\
G(z ; \mu)=C\left(z I-A_{0}-A_{1} z^{-1}-A_{2} z^{-2}\right)^{-1} B \\
=\frac{1}{z-\mu-\frac{a}{Z}-\frac{2 u}{z^{2}}}
\end{gathered}
$$

where $e(k)=-C x(k)=-x(k)$, the fixed point in the frequency domain is $\hat{e}=\frac{1-3 a}{\mu}-1$. The open-loop system matrix is

$$
G\left(e^{i \omega} ; \mu\right) J(\mu) e^{-i \omega \tau_{3}}=\frac{2 \mu-2+6 a}{e^{i \omega}-\mu-a e^{-i \omega}-2 a e^{-i 2 \omega}} e^{-i \omega \tau_{3}}
$$

with its eigenvalue

$$
\lambda\left(e^{i \omega} ; \mu\right)=G\left(e^{i \omega} ; \mu\right) J(\mu) e^{-i \tau_{3}} .
$$

Let $\hat{\lambda}(\omega)=\lambda\left(e^{i \omega} ; \mu\right)$, then the associate left and right eigenvector are $s^{T}=v=1$.

The matrices $Q$ and $L$ are

$$
Q=-2 \mu \quad L=0
$$

and the linearized close-loop function is

$$
H(z)=\frac{z^{2}}{z^{3}+(\mu-2+6 a) z^{2}-a z-2 a} .
$$

Then

$$
V_{0 H}=\frac{\mu}{2(\mu-1+3 a)}
$$

$$
V_{2 H}=\frac{\mu e^{i 4 \omega} e^{-i 2 \omega \omega_{j}}}{2\left[e^{i 6 \omega}+(\mu-2+6 a) e^{i 4 \omega}-a e^{i 2 \omega}-2 a\right]}
$$

and

$$
\xi_{H d}=-\frac{e^{i 2 \omega}}{e^{i 32}-\mu e^{i 2 \omega}-a e^{\omega \omega}-2 u} p_{H d}
$$

with

$$
\begin{aligned}
p_{H d} & =e^{-i, \pi_{3}}\left\{-\frac{\mu^{2}}{\mu-1+3 u}\right. \\
& \left.-\frac{\mu^{2} e^{i 4 \omega}}{2\left[e^{i 6 \omega}+(\mu-2+6 a) e^{i 4 \omega}-a e^{i 2 \omega}-2 a\right]}\right\} .
\end{aligned}
$$




\section{ACKNOW LEDGMENTS}

Since

$$
\hat{\lambda}(\omega)=-1+\xi_{H d} \theta^{2}
$$

can be thought as the intersection between the locus of $\hat{\lambda}(\omega)$ and a half-line starting at $-1+i 0$ in the direction defined by $\xi_{H d}(\omega)$. Denote the intersection point by $q(\hat{\omega}, \mu)=\hat{\lambda}(\hat{\omega})$, which is attained by using graphical method, then we can compute the approximate frequency $\tilde{\omega}$ and its amplitude $\theta=\left[1+q / \xi_{H d}\right]^{1 / 2}$. So the approximate expression of the invariant circle emerging from Hopf bifurcation is as follows

$$
x(k)=-e(k)=-\hat{e}-\operatorname{Re}\left\{\theta^{2} V_{0 H}+\theta v e^{i k \tilde{\omega}}+\theta^{2} V_{2 H} e^{i 2 k \tilde{\omega}}\right\} .
$$
by

The stability of the emerging periodic solution is decided

$$
\sigma_{H d}=\operatorname{Re}\left\{\frac{e^{i 3 \omega}-\mu e^{i 2 \omega}-a e^{i \omega}-2 a}{2(\mu-1+3 a)\left(-e^{i 3 \omega}-a e^{i \omega}-4 a\right)} p_{H d}\right\}
$$

Specifically, for $\tau_{3}=0$, the critical value $\mu_{0}=\frac{1}{2 a}+\frac{5}{2}-8 a$, $\cos \left(\omega_{0}\right)=-\frac{1}{4}-\frac{1}{4 a} \quad$ for $\quad a>1 / 3 \quad$, then $\sigma_{H d}>0$ for $0.3632<a<0.5$, so the closed invariant circle bifurcating from the fixed point is stable. We also have the closed invariant circle is unstable since $\sigma_{H d}<0$ when $1 / 3<a<0.3632$.

Assuming, for instance, that $\mu=0.56$ for $a=0.4$ ( $\mu_{0}=0.55$ ), the intersection point is $q=-1.012-0.001249 i$ at $\tilde{\omega}=3.7$, the locus of $\hat{\lambda}(\omega)$ and the half-line starting at $-1+i 0$ in the direction defined by $\xi_{H d}(\tilde{\omega})$. The locus intersects the half-line verifying the existence of an invariant cycle.

In the same way, we can consider the flip bifurcation (19) at the point $\hat{x}=1+\frac{3 a-1}{\mu}$ for $\mu=\mu_{0}=3-5 a(a<1 / 3)$. By computing $\quad \sigma_{D d}=\frac{\mu^{2}(1+\mu+a)}{(\mu-1+3 a)^{2}(1-3 a)}>0 . \quad$ For instance, assuming that $a=0.1$, then $\mu_{0}=2.5, \omega_{0}=\pi$ and the stable 2periodic solutions are bifurcating from the fixed point $\hat{x}=1+\frac{3 a-1}{\mu}$.

\section{CONCLUSION}

A frequency domain approach to detect Neimark-Sacker and flip bifurcations in the unified formulation for discrete-time systems with multiple time-delays has been discussed by using harmonic balance approach. And the expressions that predict the emerging periodic solution and determine the stability of the periodic solution have been derived. An extension to deal with bifurcation control is very attractive in the future since FDA for maps provides a useful graphical interpretation.
This research is supported by Guangdong Philosophy and Social Science Planning Project (No. GD13XGL41) and Innovative School Project in Higher Education of Guangdong, China (No. GWTP-SY-2014-02).

\section{REFERENCES}

[1] J. L. Moiola and G. Chen, "Frequency domain approach to computation and analysis of bifurcations and limit cycles: a tutorial," Int J Bifurcation and Chaos, vol. 3, pp. 843-867, 1993.

[2] J. L. Moiola and G. Chen, Hopf Bifurcation Analysis: A Frequency Do main Approach. Singapore: World Scientific, 1996.

[3] F. S. Gentile, J. L. Moiola and E. E. Paolini, "On the study of bifurcations in delay-differential equations: a frequency-domain approach,” Int J Bifurcation and Chaos, vol. 22, pp. 99-113, 2012.

[4] T. Detroux, L. Renson, L. Masset and G. Kerschen, "The harmonic balance method for bifurcation analysis of large-scale nonlinear mechanical systems," Computer Methods in Applied Mechanics and Engineering, vol. 296, pp. 18-38, 2015.

[5] Y. Chang, X. Wang and D. Xu, "Bifurcation Analysis of a Power System Model with Three Machines and Four Buses," Int J Bifurcation and Chaos, vol. 26, 1650082, 2016.

[6] M. B. D'amico, J. L. Moiola and E. E. Paolini, "Controlling bifurcations in maps via a frequency-domain approach," Dyn Contin Discrete Impuls Syst Ser B Appl Algorithms, vol. 10, pp. 781-798, 2003.

[7] M. B. D'amico, J. L. Moiola and E. E. Paolini. "Hopf Bifurcation for maps: A frequency-doma in approach," IEEE Trans Circuits Syst I, vol. 49, pp. 281-287, 2002.

[8] M. B. D'amico, J. L. Moiola and E. E. Paolini. "Stability analysis of degenerate Hopf bifurcations for discrete-time systems," Lat in A merican Applied Research, vol. 33, pp. 413-418, 2003.

[9] L. Zeng, Y. Zhao, W. Sun and X. J. Zong. "Bifurcation of nonlinear maps with time delay in the frequency domain with application to a metapopulation model," Acta Universitatis Sun Yatseni (Scientiarum Naturaliu m), vol. 45, pp. 16-20, 2006.

[10] A. I. Mees and L. O. Chua, "The Hopf bifurcation theorem and its applications to nonlinear oscillations in circuits and systems," IEEE Trans Circuits Syst, vol. 26, pp. 235-254, 1979.

[11] D. G. Aronson, M. A. Chory, G. R. Hall and R. P. Mcgehee, "Bifurcations from an invariant circle for two-parameter families of maps of the plane: A computer-assisted study," Comm Math Phys, vol. 83, pp. 303-354, 1982.

[12] B. B. Peckham and I. G. Kevrekid is, "Period doubling with higher-order degeneracies,” SIAM J Math Anal, vol. 22, pp. 1552-1574, 1991. 